\title{
Ultra-Wideband Indoor Channel Modelling Using Ray-Tracing Software for through-the-Wall Imaging Radar
}

\author{
Christophe Lièbe, ${ }^{1}$ Pierre Combeau, ${ }^{2}$ Alain Gaugue, ${ }^{1}$ Yannis Pousset, ${ }^{2}$ Lilian Aveneau, ${ }^{2}$ \\ Rodolphe Vauzelle, ${ }^{2}$ and Jean-Marc Ogier ${ }^{1}$
}

${ }^{1}$ Laboratoire Informatique Image Interaction (L3I), EA 2118, Université de La Rochelle, Av M. Crépeau,
17042 La Rochelle Cedex 01, France
${ }^{2}$ Xlim-SIC (Signal Image Communication), UMR CNRS 6172 SP2MI, Bd Marie et Pierre Curie, BP 30179,
86962 Futuroscope Chasseneuil Cedex, France

Correspondence should be addressed to Alain Gaugue, alain.gaugue@univ-lr.fr

Received 22 March 2010; Accepted 2 June 2010

Academic Editor: Shyh-Kang Jeng

Copyright (C 2010 Christophe Lièbe et al. This is an open access article distributed under the Creative Commons Attribution License, which permits unrestricted use, distribution, and reproduction in any medium, provided the original work is properly cited.

\begin{abstract}
This paper presents a new software for design of through-the-wall imaging radars. The first part describes the evolution of a ray tracing simulator, originally designed for propagation of narrowband signals, and then for ultra-wideband signals. This simulator allows to obtain temporal channel response to a wide-band emitter ( $3 \mathrm{GHz}$ to $10 \mathrm{GHz}$ ). An experimental method is also described to identify the propagation paths. Simulation results are compared to propagation experiments under the same conditions. Different configurations are tested and then discussed. Finally, a configuration of through-the-wall imaging radar is proposed, with different antennas patterns and different targets. Simulated images will be helpful for understanding the experiment obtained images.
\end{abstract}

\section{Introduction}

UWB technology provides a number of unique operational capabilities in a diverse range of applications, especially in the fields of high-resolution radars and high-rate communication systems. One of the best applications of UWB radar is the "through-wall vision." This technology can be used by rescue services, searching for people in rubble and in buildings on fire. The military industry can use this technology for bomb disposal, neutralising of aggressors, and hostage rescue, and so forth.

UWB radar presents many advantages compared to other systems. UWB for short range radar includes extremely fine range resolution (could be subcentimeter), high power efficiency (because of low transmit duty cycle), noise robustness and low probability of detection (due to large bandwidth), ability to detect moving or stationary targets and possibility of identifying them, and the ability to penetrate wall and floor materials to "see through" (concrete, up to $5 \mathrm{GHz}$, bricks up to $10 \mathrm{GHz}$ and "light" walls up to $60 \mathrm{GHz}$ ).
There are less than ten laboratories and companies throughout the world, which work on the UWB radar application "detection/vision through the walls." Company Time Domain (USA) was the first producer of this system. Company Camero (Israel) created a radar (Xaver 800), which currently has the best performances. These systems use classical MTI filter [1] to extract the relevant information. This technique highlights the moving target but erases all echoes emitted (or resulting) from the environment. This phenomenon is illustrated by the following experiment.

A temporal response from a corridor acquired through a concrete wall by our UWB radar [2] is shown on Figure 1. The scene (cf. Figure 1(a)) contains a moving human with a closed door behind. Figure 1(b) (and Figure 1(c)) is an acquisition obtained in one direction up $t_{0}$ (and up $t_{0}+500 \mathrm{~ms}$, resp.). Figure $1(\mathrm{~d})$ is the result from a basic MTI algorithm applied at these signals. The moving target ("E4" echo) is clearly identified with this MTI filter, but all other echoes disappear. Unfortunately, these other echoes (or reverberation "echoes"), as example echoes E1, E2, or E3 


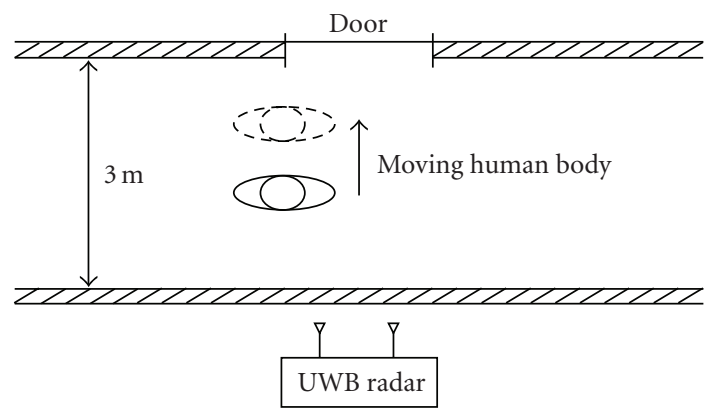

(a)

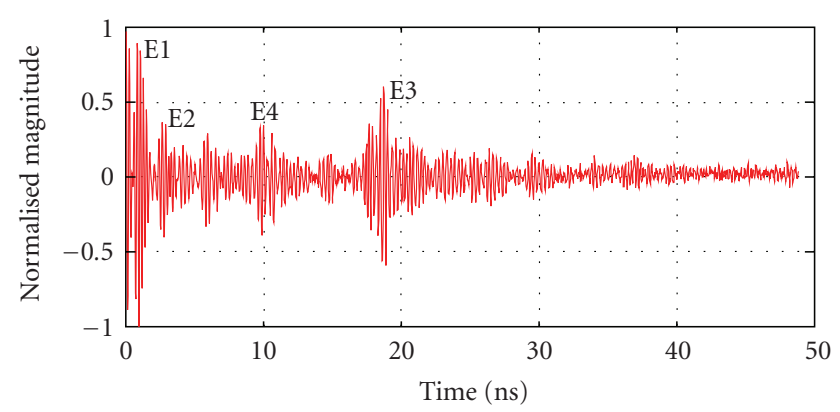

(b)

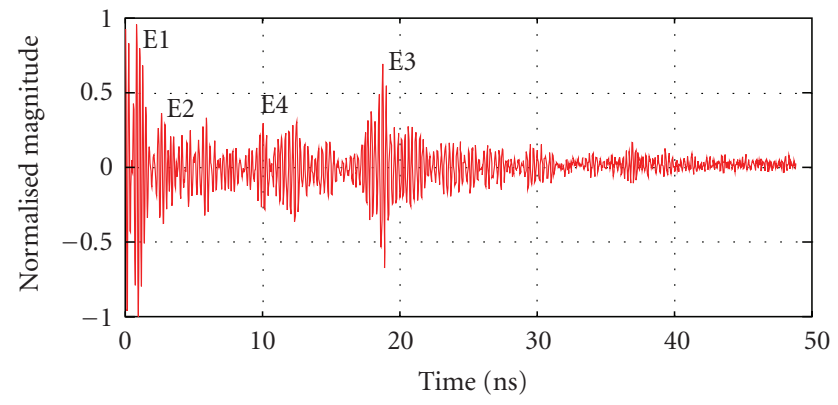

(c)

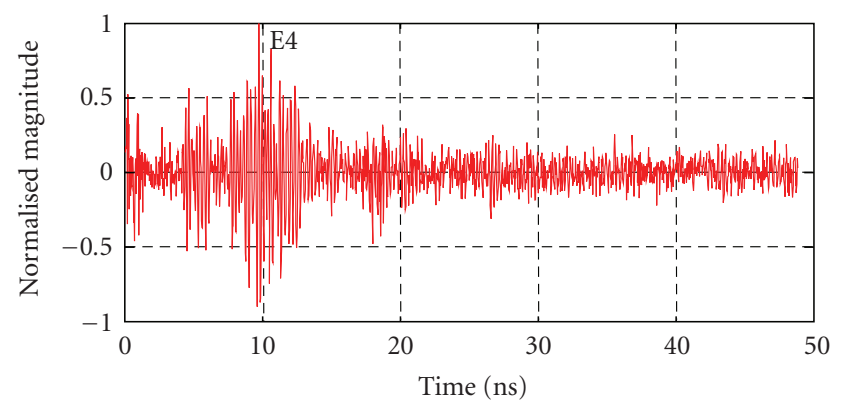

(d)

FIGURE 1: Temporal response acquired through a concrete wall: (a) description of the scene, (b) correspond to an acquisition at $t_{0}$, (c) correspond to an acquisition at $t_{0}+500 \mathrm{~ms}$, and (d) subtraction between (b) and (c) to clearly identify the "E4" echo corresponding to the moving target.

in Figure 1(b), contain a lot of information about the scene environment (geometry of the room, presence of furniture, etc.). But in practice it is very difficult to find their causes. For example in our scene, the "E2" echo is due to the reflection from the wall, and the "E3" echo corresponds to a wave reflected by the door. These signals show the possibility of recovering complementary information other than the presence or absence of a human body located behind a wall.

These reverberation "echoes" result from multiple reflection of the signal transmitted by walls. The room reverberation is similar to the sound reverberation at these wavelengths and is characterized by long ringing nature. If we can analyse each reverberation, we can extract significant parameters to reconstruct the geometry of the room (length, height, width) situated behind the wall. Concretely, an experimental approach does not allow identification and interpretation of all these echoes. Thus, we have also developed a 3D wave propagation simulator to predict and analyse each reverberation echo.

Designing a through-the-wall radar imaging (TWRI) is a complex and difficult problem that requires crossdisciplinary research in a large field (antennas and array processing, image processing, etc.). But to gain knowledge, the propagation characterization of UWB signal is vital for optimal design and accurate evaluation of an UWB imaging radar. Many researchers have studied the propagation of electromagnetic waves and many methods are applied to simulate TWRI. The most classical is based on the finitedifference time domain (FDTD) method [3]. In this paper we propose to use a model based on the ray optics to predict specific propagation properties of UWB waves in a room. This model will be the heart of a real simulator of a TWRI.

\section{The Propagation Simulator}

As indicated in the previous section, some propagation simulators have been used in literature to analyse received waves and identify the environment impact on their propagation for TWRI applications. Most of them are based on exact numerical methods like Finite Difference in Time Domain (FDTD) [4] which is able to achieve very accurate propagation simulation but often implies prohibitive computation time in real environment, as it is so vast. Indeed, its use necessitates the resolution of Maxwell equations on each voxel of a 3D spatial digitisation of the treated scene. The size of each voxel is about a tenth of a wavelength and so there is no computational solution at our frequencies of interest for TWRI, that is, in the $3-10 \mathrm{GHz}$ band, for which more than $10^{9}$ voxels are necessary for an environment of $70 \mathrm{~m}^{3}$ at a single frequency of $10 \mathrm{GHz}$.

Bearing this in mind, a realist solution for treating complex environments is to use an asymptotic method associated to ray-tracing algorithm. In [5], Shargo and Melody present a method using ray-tracing to correct defocusing effects appearing in SAR imagery between $500 \mathrm{MHz}$ and $1.5 \mathrm{GHz}$. Nevertheless, to the author's knowledge, the raytracing potential has not been clearly shown in the studied context. So, in this article we propose to show that it is possible to rebuild geometrical properties of an environment from information provided by ray-tracing based on our propagation simulator in the $3-10 \mathrm{GHz}$. The architecture of which is presented in this section. 


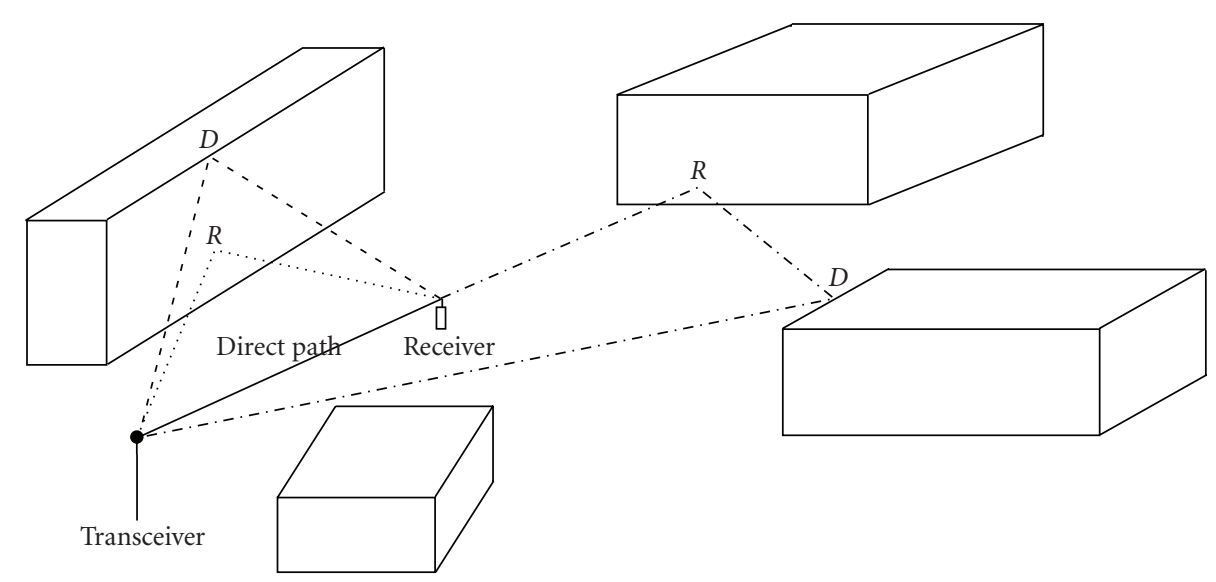

FIGURE 2: Multipaths phenomenon: $R$ is a reflexion point, and $D$ is a diffraction one.

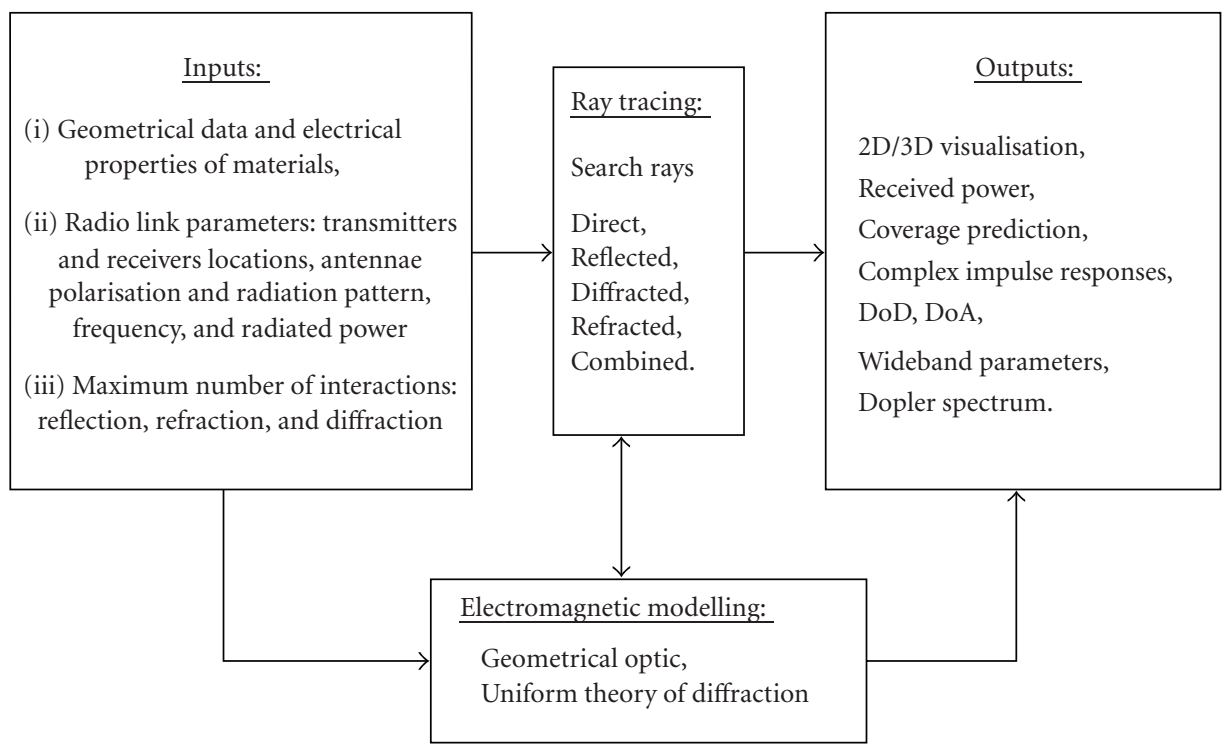

FIGURE 3: RAPSOR's architecture.

2.1. The Multipath Phenomenon. The multipath phenomenon governs the wave propagation in the studied frequency bandwidth (cf. Figure 2).

It is a fact that, during their propagation, waves move along different paths and interact with environment obstacles according to physical phenomena such as reflection, refraction, and diffraction. These interactions can affect the propagation direction, the amplitude, phase and polarization of the transmitted wave. Therefore the received signal results from the combination of all the waves having followed specific paths from the transmitter.

2.2. The Simulator's Architecture. In this context, we have developed within the Xlim-SIC laboratory a RAy Propagation SimulatOR (RAPSOR) (http://rapsor.sourceforge.net/) allowing prediction of the multipath phenomena and their electromagnetic characteristics. The flow chart on Figure 3 illustrates the RAPSOR architecture.
This last one is divided in four main parts, inputs and outputs which are presented in Section 2.5, the electromagnetic modelling based on Geometrical Optics (GO) laws extended to the Uniform Theory of Diffraction (TUD) described in Section 2.4, and the ray-tracing algorithm for path determination which is the subject of the next section.

2.3. Ray-Tracing Algorithm. Two different techniques are used to, respectively, treat reflected/transmitted and diffracted rays. Reflected rays are determined by the sourceimage technique which is illustrated in Figure 4.

In this simple case, the image $\mathrm{Tx}_{1}$ of source $\mathrm{Tx}$ with regards to $\mathrm{Wall}_{1}$ is first computed, then image $\mathrm{Tx}_{2}$ of $\mathrm{Tx}_{1}$ with regards to Wall 2 . Connecting $\mathrm{Tx}_{2}$ to $R x$, we can determine a reflected point $P_{2}$. In the same way, another reflection point $P_{1}$ is defined by the intersection between Wall $l_{1}$ and line $\left(\mathrm{TxTx}_{1}\right)$. 


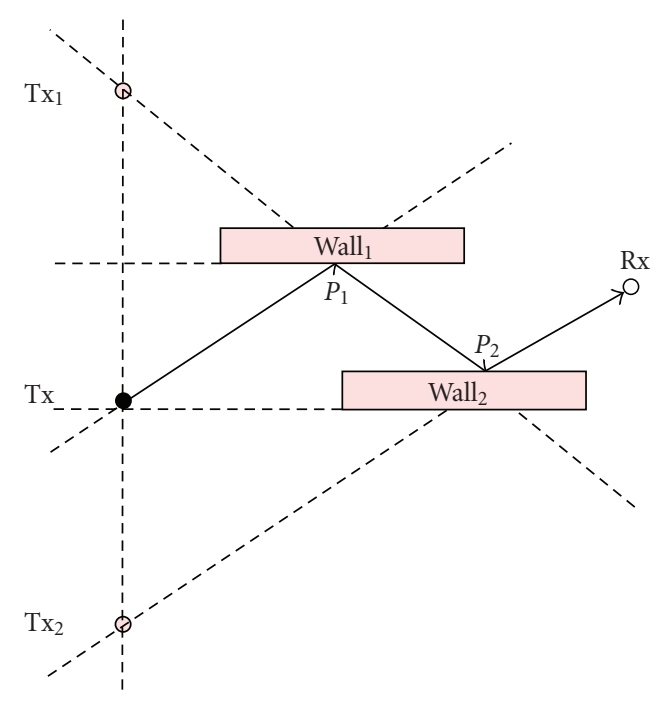

FIGURE 4: Source image method.

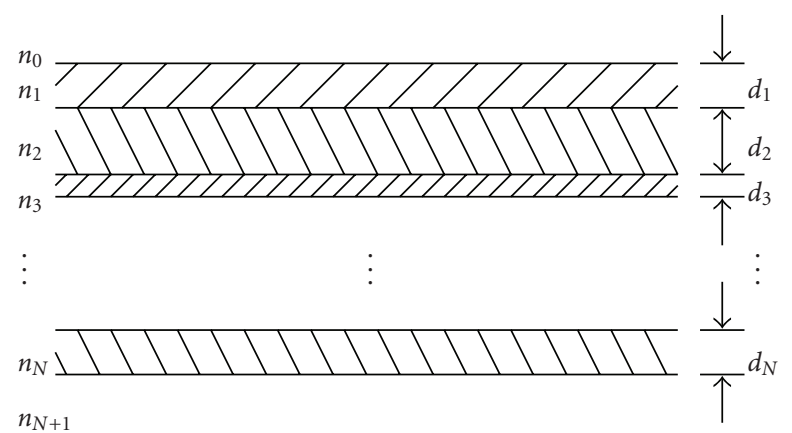

Figure 5: Multilayered structure. $n_{i}$ and $d_{i}$ are the complex refractive index and the thickness of the $i$ th layer, respectively.

Determination of a transmitted ray through a wall simply consists in continuing the incident ray when this one strikes an obstacle, which is a choice proposed and validated in [6].

Finally, diffracted rays are computed using an iterative algorithm based on generalized Fermat principle [7].

2.4. Electromagnetic Modelling. The reflected, refracted, and diffracted electrical fields are expressed in matrix form according to perpendicular and parallel components relative to incidence plane:

$$
\left[\begin{array}{ll}
E_{/ l}^{r, t, d} & (r) \\
E_{\perp}^{r, t, d} & (r)
\end{array}\right]=\left[\begin{array}{cc}
R, T, D_{/ /} & 0 \\
0 & R, T, D_{\perp}
\end{array}\right]\left[\begin{array}{ll}
E_{/ /}^{i} & (0) \\
E_{\perp}^{i} & (0)
\end{array}\right] \widetilde{A}(r)
$$

where $r$ represents the curvilinear abscissa along the ray, $E_{, /,}^{r, t, d}$ and $E_{\perp}^{r, t, d}$, respectively, the parallel and perpendicular components of reflected/transmitted/diffracted electrical fields, $R, T, D_{/ /, \perp}$ the parallel/perpendicular reflection/transmission/diffraction coefficients, and $\widetilde{A}(r)$ the complex divergence coefficient connected to the energy conservation in a ray tube [8].
Furthermore, the reflection and transmission coefficients take into account the multilayered structure of the walls according to Figure 5.

Their expressions are given in $[9,10]$ :

$$
\begin{aligned}
& R=R_{0,1}^{\prime}, \\
& T=T_{0,1}^{\prime},
\end{aligned}
$$

where

$$
\begin{aligned}
& R_{i, i+1}^{\prime}= \begin{cases}\frac{R_{i, i+1}+R_{i+1, i+2}^{\prime} e^{-j 2 \delta_{i+1}^{(1)}}}{1+R_{i, i+1} R_{i+1, i+2}^{\prime} e^{-j 2 \delta_{i+1}^{(1)}}}, & 0 \leqslant i \leqslant N-1 \\
R_{N, N+1}, & i=N,\end{cases} \\
& T_{i, i+1}^{\prime}= \begin{cases}\frac{T_{i, i+1} T_{i+1, i+2}^{\prime} e^{-j \delta_{i+1}^{(2)}}}{1+R_{i, i+1} R_{i+1, i+2}^{\prime} e^{-j 2 \delta_{i+1}^{(1)}}}, & 0 \leqslant i \leqslant N-1 \\
T_{N, N+1}, & i=N .\end{cases}
\end{aligned}
$$

In (3)

$$
\begin{gathered}
\delta_{i}^{(1)}=\frac{2 \pi d_{i}}{\lambda} \sqrt{n_{i}^{2}-\sin ^{2}(\theta)}, \\
\delta_{i}^{(2)}=\frac{2 \pi d_{i}}{\lambda}\left(\sqrt{n_{i}^{2}-\sin ^{2}(\theta)}-\cos (\theta)\right) .
\end{gathered}
$$

In these equations, $\lambda$ is the wavelength in free space, $d_{i}$ is the thickness of the $i$ th layer, $N$ is the number of layers, $n_{i}$ is the complex refractive index of the $i$ th layer, and $\theta$ is the angle of incidence.

Furthermore, $R_{i j}$ and $T_{i j}$ are given in

$$
\begin{gathered}
R_{\perp i, i+1}=\frac{\sqrt{n_{i}^{2}-\sin ^{2}(\theta)}-\sqrt{n_{i+1}^{2}-\sin ^{2}(\theta)}}{\sqrt{n_{i}^{2}-\sin ^{2}(\theta)}+\sqrt{n_{i+1}^{2}-\sin ^{2}(\theta)}}, \\
R_{/ / i, i+1}=\frac{n_{i+1}^{2} \sqrt{n_{i}^{2}-\sin ^{2}(\theta)}-n_{i}^{2} \sqrt{n_{i+1}^{2}-\sin ^{2}(\theta)}}{n_{i+1}^{2} \sqrt{n_{i}^{2}-\sin ^{2}(\theta)}+n_{i}^{2} \sqrt{n_{i+1}^{2}-\sin ^{2}(\theta)}}, \\
T_{\perp i, i+1}=\frac{2 \sqrt{n_{i}^{2}-\sin ^{2}(\theta)}}{\sqrt{n_{i}^{2}-\sin ^{2}(\theta)}+\sqrt{n_{i+1}^{2}-\sin ^{2}(\theta)}}, \\
T_{/ / i, i+1}=\frac{2 n_{i} n_{i+1} \sqrt{n_{i}^{2}-\sin ^{2}(\theta)}}{n_{i+1}^{2} \sqrt{n_{i}^{2}-\sin ^{2}(\theta)}+n_{i}^{2} \sqrt{n_{i+1}^{2}-\sin ^{2}(\theta)}},
\end{gathered}
$$

where $R_{\perp i, i+1}$ and $R_{/ / i, i+1}$ are Fresnel's reflection coefficients for the interface between the $i$ th and the $i+1$ th dielectric media when the electric field is, respectively, perpendicular and parallel to the plane of incidence. $R_{\perp i, i+1}$ and $R_{/ / i, i+1}$ are substituted for $R_{i, i+1}$ in the cases of perpendicular and parallel polarization, respectively.

Finally, diffracted coefficients are computed according to the TUD formulation given by Kouyoumjian and Pathak in [8] and then extended to the case of dielectric media by Luebbers in [11]:

$$
D_{/ /, \perp}\left(L, \phi, \phi_{0}, n\right)=D_{1}+D_{2}+R_{/ /, \perp}\left(D_{3}+D_{4}\right),
$$




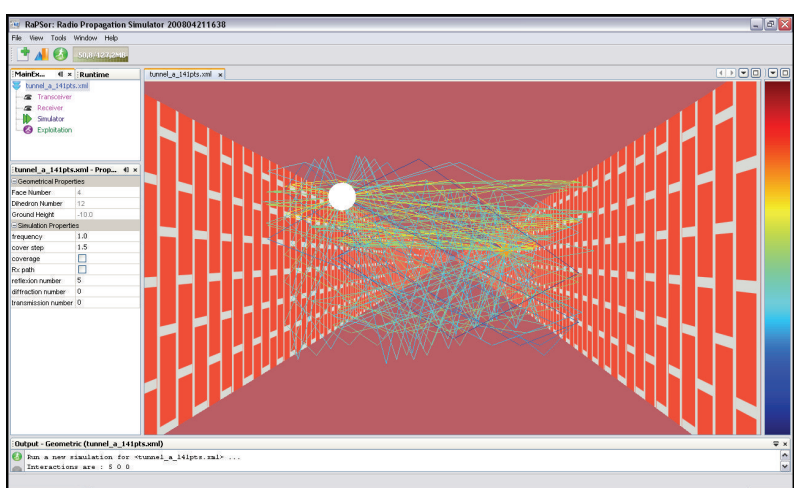

(a)

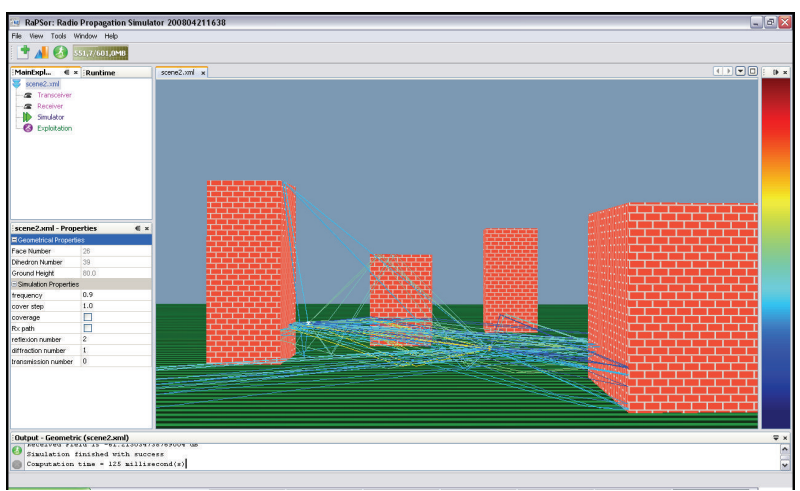

(c)

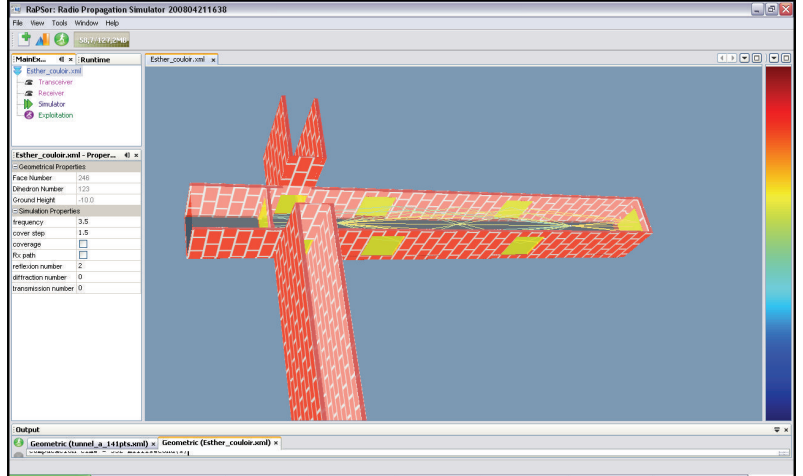

(b)

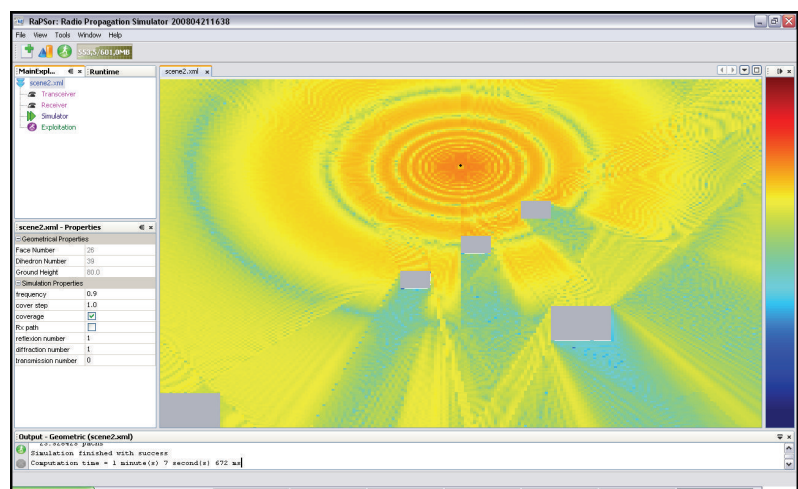

(d)

FIGURE 6: RAPSOR interfaces: 3D paths in (a) tunnel environment, (b) indoor environment, (c) outdoor environment, and (d) is coverage area in outdoor environment.

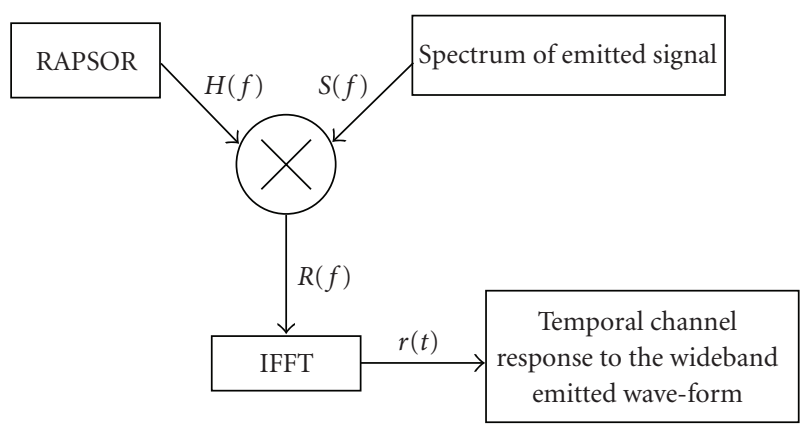

Figure 7: Computation chart of the channel response to a wideband wave-form.

where

$$
\begin{aligned}
D_{1}= & \frac{-e^{-j(\pi / 4)}}{2 n \sqrt{2 \pi k} \sin \beta_{0}} \cot \left[\frac{\pi+\left(\phi-\phi_{0}\right)}{2 n}\right] \\
& \times F\left[k L a^{+}\left(\phi-\phi_{0}\right)\right] \\
D_{2}= & \frac{-e^{-j(\pi / 4)}}{2 n \sqrt{2 \pi k} \sin \beta_{0}} \cot \left[\frac{\pi-\left(\phi-\phi_{0}\right)}{2 n}\right] \\
& \times F\left[k L a^{-}\left(\phi-\phi_{0}\right)\right]
\end{aligned}
$$

$$
\begin{aligned}
D_{3}= & \frac{-e^{-j(\pi / 4)}}{2 n \sqrt{2 \pi k} \sin \beta_{0}} \cot \left[\frac{\pi+\left(\phi+\phi_{0}\right)}{2 n}\right] \\
& \times F\left[k L a^{+}\left(\phi+\phi_{0}\right)\right], \\
D_{4}= & \frac{-e^{-j(\pi / 4)}}{2 n \sqrt{2 \pi k} \sin \beta_{0}} \cot \left[\frac{\pi-\left(\phi+\phi_{0}\right)}{2 n}\right] \\
& \times F\left[k L a^{-}\left(\phi+\phi_{0}\right)\right] .
\end{aligned}
$$

The transition function $F$ is the modified Fresnel integral:

$$
F(x)=2 j \sqrt{x} e^{j x} \int_{\sqrt{x}}^{\infty} e^{-j t^{2}} d t
$$

where variable $x$ depends on parameters $k, L, a^{ \pm} . k$ is the wave vector, $L$ is a distance parameter depending on wave nature, and $a$ depends on the diffracting object shape and on incident/diffracted angles, respectively, noted by $\phi_{0}$ and $\phi$.

2.5. Simulator Inputs/Outputs. As we can see in Figure 3, inputs are globally divided in three types.

(i) The first one is the $3 \mathrm{D}$ geometrical description of the environment based on plane polygons. Particularly, for outdoor environments, this description 


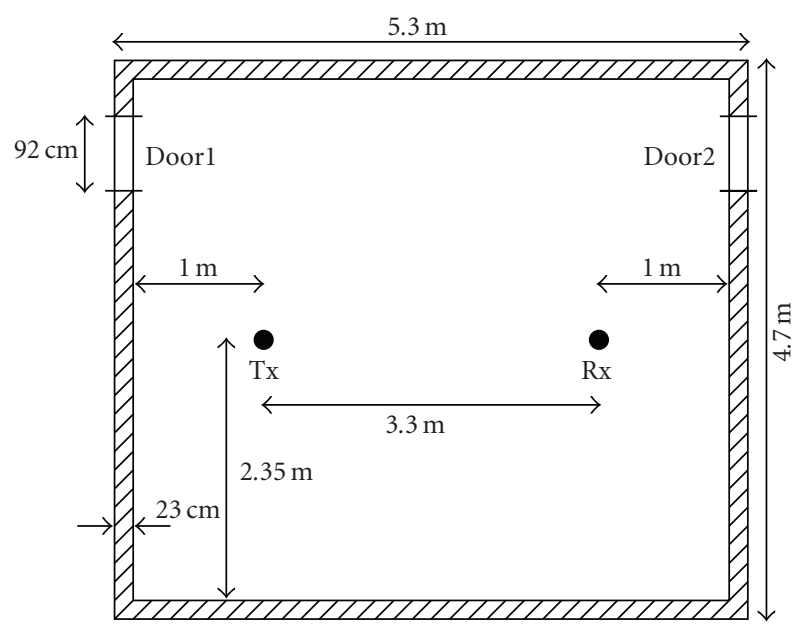

FIGURE 8: Experiment room with position of the transceiver (Tx) and the receiver $(\mathrm{Rx})$ for temporal response measurement.

is obtained by terrain data based achieved by the Institut Geographique National (IGN) in France or another similar map maker. In an indoor environment, we use architectural databases like DXF (Autocad) or data obtained by a 3D model maker. Obviously, this geometrical description is completed with an electrical one which details permittivity and conductivity of materials composing the environment, parameters whose values depend on the frequency.

(ii) The second is the radio link parameters, as transceiver(s)/receiver(s) location, antenna radiation pattern and polarization, frequency, and emitted power.

(iii) The third is the simulation parameters in terms of maximum interactions number. As shown in the previous section, modelled interactions are visibility, reflection, refraction, and diffraction.

Concerning outputs, RAPSOR provides complex 3D electrical fields for each path reaching the receiver. Consequently, complete and fine channel characterization is achievable, in the form of complex impulse response, power delay profile and all wideband parameters (RMS delay spread, etc.), Doppler spectrum, received power and coverage area, for example. Furthermore, the important information is the visualization in 3D of all the paths going from the transceiver to the receiver in the simulated environment, as shown in Figure 6, which illustrates the 3D interface of RAPSOR. This is very useful to understand the propagation phenomena as we will see in Section 4 .

2.6. From Narrow to Wideband. As we have seen in Section 1, the TWRI method presented in this article is based on a wideband radio channel analysis, between 3 and $10 \mathrm{GHz}$. In particular, we have to compute the frequency response of the channel in this band of frequencies. Since electrical field computation achieved by asymptotic methods is only valid at one frequency, it is necessary to compute the field for each frequency of the considered band. Thus, from one frequency to the next, one can obtain the frequency response of the channel $H(f)$.

Nevertheless, the greater the frequency band is, the longer the computation time will be. So, to stay within reason, we have developed a technique consisting in memorising the geometrical ways of each path (ray tracing result). Then we just have to recompute the electrical field corresponding to each path, without processing to the ray tracing step, which is the most time consuming in a propagation simulation. Please note that, in our simulations, we consider for each frequency the corresponding 3D radiation pattern of used antennas.

Finally, computing the temporal response of the studied environment to a wideband wave-form is simply resumed in the schema in Figure 7.

Considering $H(f)$ and $S(f)$, respectively, the wideband frequency response of the radio channel and the emitted signal spectrum, the spectrum of the received signal is

$$
R(f)=S(f) \cdot H(f) .
$$

The temporal channel response of the wideband-emitted wave-form $r(t)$ is so obtained by the Inverse Fourier Transform (IFFT) of $R(f)$. Thus, $r(t)$ will to be compared to the received signal of experimental measurement platform presented in Section 4.

\section{Experimental Setup}

In this section we describe the test scene and UWB signal used to acquire experimental results to compare with the RAPSOR simulator. All parameters described in this section are those used for simulation (cf. Section 4).

3.1. Experimental Platform. The experiment takes place in a room (cf. Figure 8) of $5.3 \times 4.7 \mathrm{~m}^{2}$, with a ceiling $3 \mathrm{~m}$ high. Walls, floors, and ceilings are made of concrete or concrete blocks (dielectric constant $\varepsilon_{r}=9$ or $\varepsilon_{r}=2.45$ ). Floor and ceiling have a thickness equal to $30 \mathrm{~cm}$ and walls $23 \mathrm{~cm}$, only. The two doors are in plywood (dielectric constant $\varepsilon_{r}=3.55$ ).

3.2. UWB Signal. The transmitter used to generate a UWB pulse is manufactured by Time Domain Corporation [12] (PulsON 200 EVK). The emitted pulse is a sinusoidal signal modulated by a Gaussian pulse with a central frequency at $4.7 \mathrm{GHz}$ and a bandwidth at $3.2 \mathrm{GHz}$ (cf. Figure 9). To radiate, the transmitter and the receiver (also a PulsON 200 $\mathrm{EVK}$ ) use an omnidirectional antenna with a $3 \mathrm{~dB}$ gain.

3.3. Temporal Response of the Room. To measure the pulse response of the room, we place the emitter and the receiver in the room, as shown in Figure 8. Doors are closed and operators leave the room. Nothing and no one is present in the room; both the emitter and receiver are controlled by a personal computer through an Ethernet cable. Temporal response of this configuration is shown in Figure 10. This acquisition is used to be compared to simulation results. 


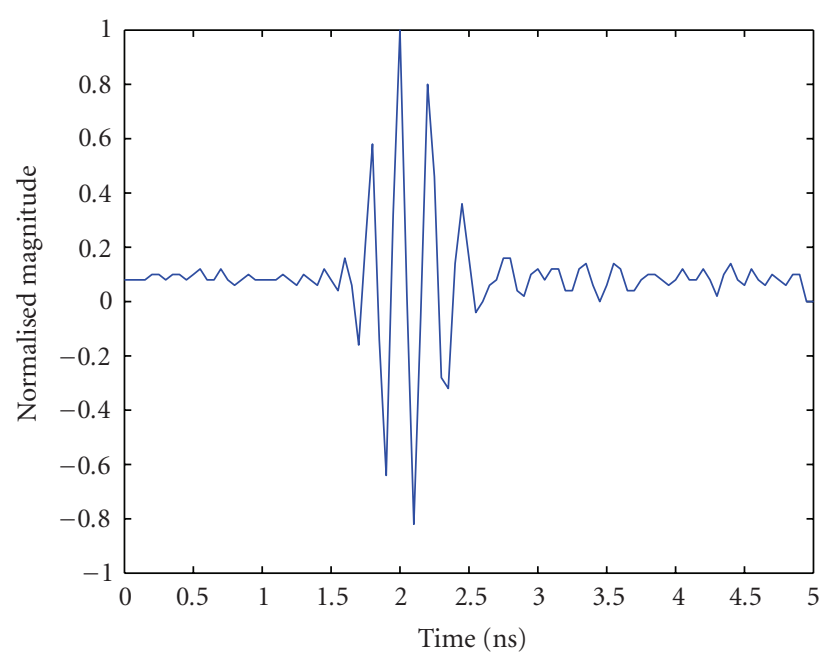

(a)

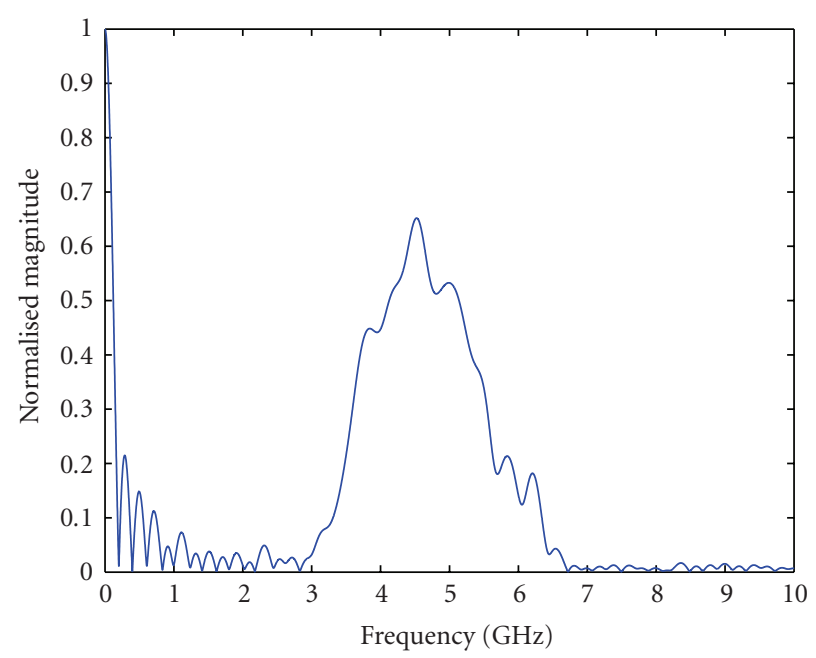

(b)

FIgURE 9: Temporal wave-form (a), and Wave-form spectrum obtained by FFT (b). The pulse width is around 1 nanosecond and the bandwidth is comprised of $3 \mathrm{Ghz}$ up to $6.2 \mathrm{Ghz}$.

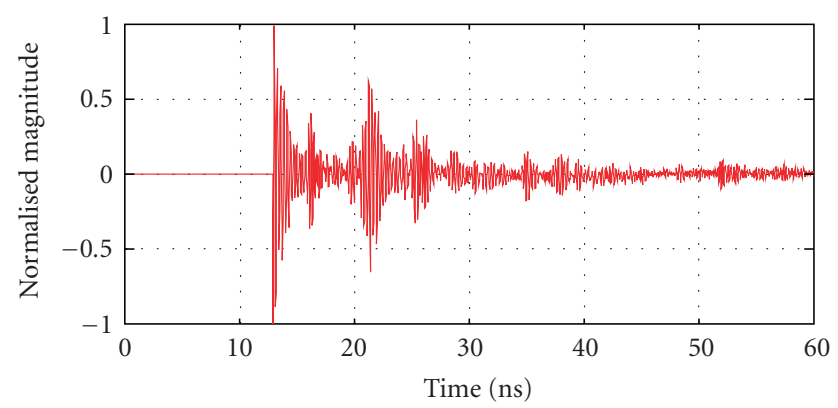

Figure 10: Temporal response of the experiment room in symmetric case (cf. Figure 8).

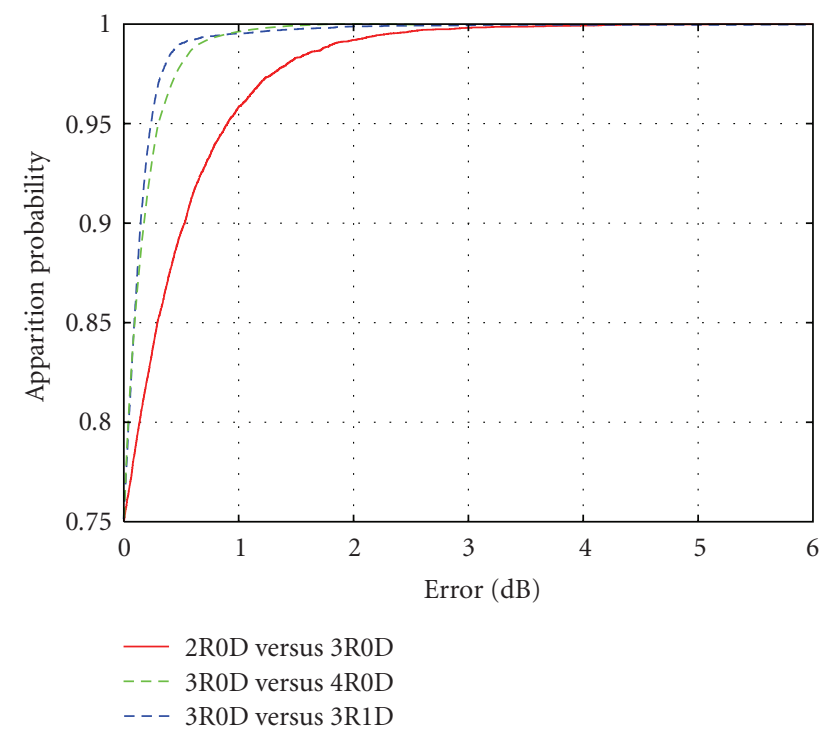

FIGURE 11: Error cumulative function of estimated received power for different cases of reflection and diffraction.

\section{Test Room Simulation}

In this section we first present the simulation driven to the temporal channel response to wideband wave-form of Figure 9(a). Then, we analyse this simulated signal by explaining the propagation phenomena and linking these phenomena to the different echoes of a received signal.

As we have seen in Section 2.6, the temporal channel response to the used wideband wave-form is obtained by application of Figure 7 algorithm. From this algorithm, we first have to compute received power at each frequency of the [ $3 \mathrm{GHz}, 10 \mathrm{GHz}$ ] band and, doing this, the channel frequency response $H(f)$ of Figure 7.

But beforehand, the optimal combination of interactions (reflection, transmission, and diffraction) that have to be take into account into simulations has to be determined and this is the purpose of the next section.

4.1. Optimal Interactions Combination. This one corresponds to the best compromise between simulation accuracy and computation time. Indeed, the higher the interactions combination is, the more accurate the simulation will be but also the longer the computation time will be. To reach this compromise, Figure 11 presents different curves of error cumulative function of estimated received power, for different interactions combinations. These curves have been computed from the coverage areas of Figure 8's $T_{x}$ according to the interactions combination. These last ones have been computed on a regular grid of $5 \mathrm{~cm}$ spaced receivers, which corresponds to 9934 receivers.

From Figure 11, the red curve (2R0D versus 3R0D) represents the error cumulative function of received power between the considered 3 and 2 reflections. One can see that $95 \%$ of errors are less than $0.8 \mathrm{~dB}$. On the blue curve (3R0D versus 3R1D) which represents the same information between the considered 3 and 4 reflections, one can observe 


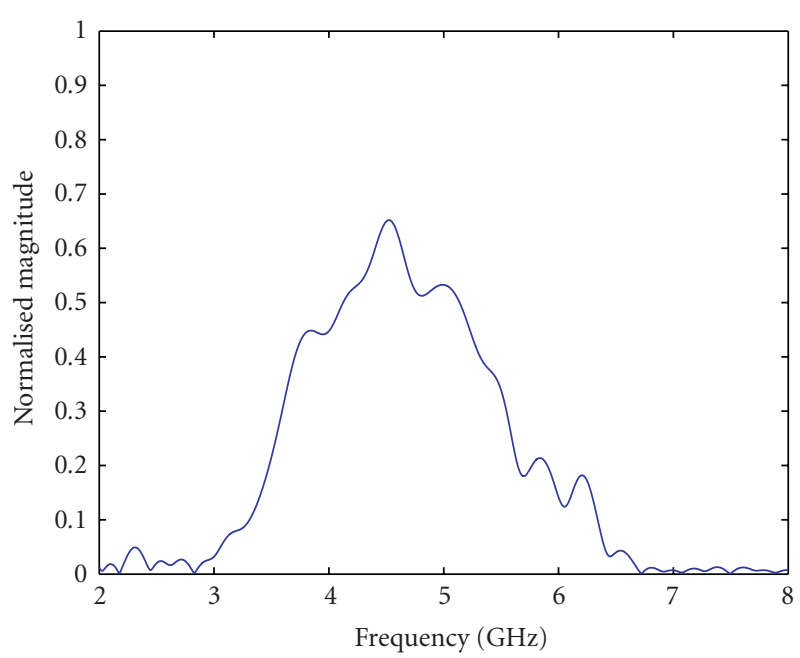

(a)

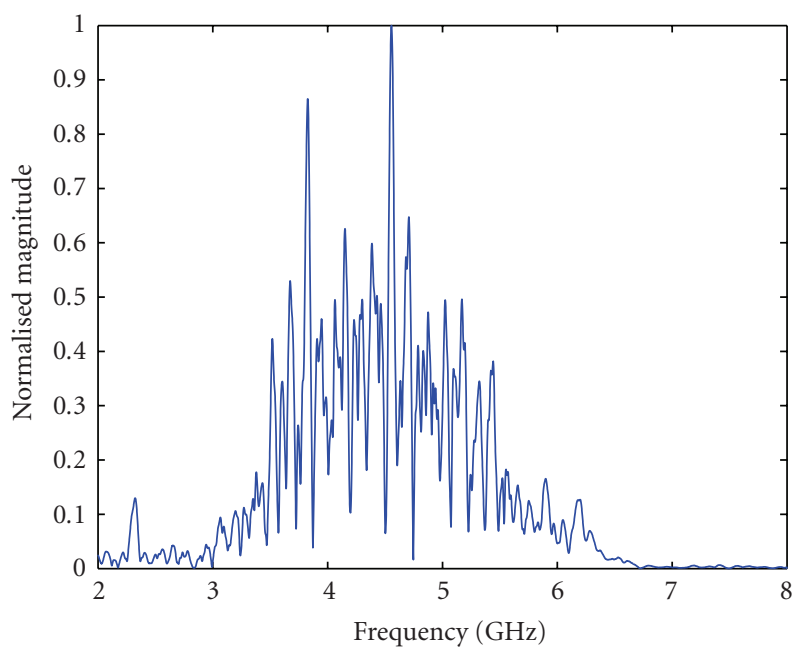

(c)

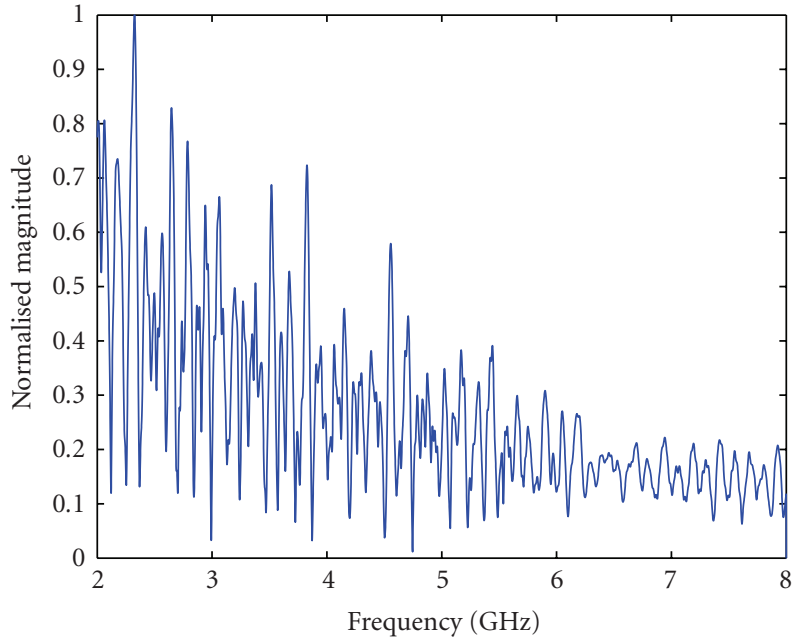

(b)

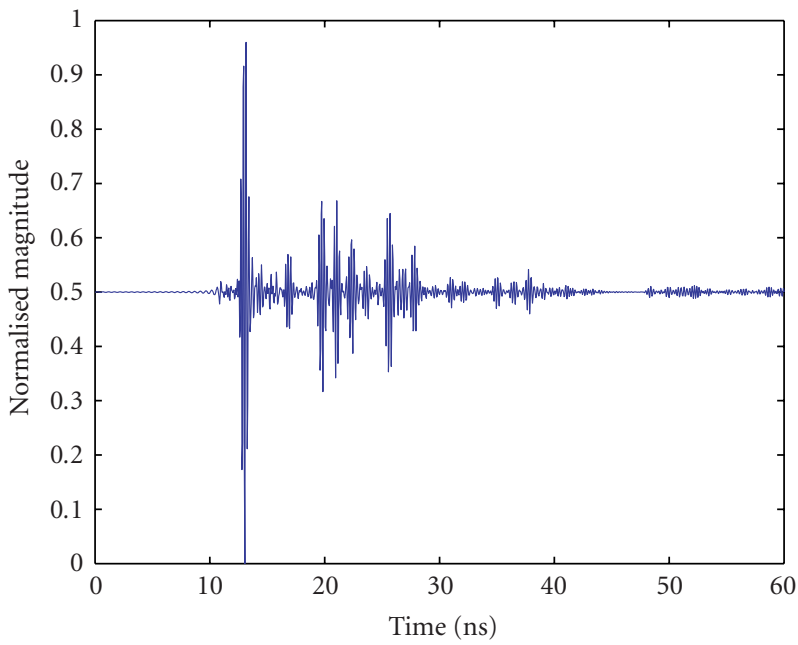

(d)

FIgURE 12: Signals in Figure 7 algorithm: (a) is the signal spectrum $S(f)$, (b) is the frequency response of the channel $H(f)$, (c) is The wideband frequency response of the radio channel $R(f)$, (d) is the temporal channel response to the wideband emitted wave-form $r(t)$.

that $95 \%$ are less than $0.3 \mathrm{~dB}$. On this last remark, one concludes that a fourth reflection does not bring significant contribution to received power, and so from here, we will only consider 3 reflections in all presented simulations.

The last curve (3R0D versus 3R1D) represents the error cumulative function of received power between considering just 3 reflections on one side, and 3 reflections plus 1 diffraction on the other side. One notices that the blue and green curves are very closed; so it appears that, in this environment, contributions corresponding to diffracted paths are nonsignificant. Conclusion of this study: from now on, we will only consider 3 reflections in all simulations.

4.2. Computation of Channel Temporal Response. Since the optimal interactions combination has been determined, we now are able to implement the algorithm of Figure 7.
Simulated $H(f)$ in the symmetric case of Figure 8, and computed with 3 reflections, is depicted in Figure 12(b).

Notice that to simulate $H(f)$, we have chosen a frequency step of $1 \mathrm{MHz}$. This choice consequently leads to a temporal observation window of $1 \mu \mathrm{s}$ which is sufficient to guarantee that all significant contributions are considered in indoor configuration [6]. Furthermore, signal processing techniques like zero-padding have been used on $S(f)$ to obtain the same frequency step as $H(f)$.

According to Figure 7, the multiplication between $S(f)$ (cf. Figure 12(a)) and $H(f)$ (cf. Figure 12(b)) provides $R(f)$ depicted in Figure 12(c).

Finally, the channel temporal response to the emitted wave-form in Figure 9(a) is obtained by Inverse Fourier Transform (IFFT) and is presented in Figure 12(d). Once again, zero-padding technique has been used on $R(f)$ to obtain the same temporal step as the measured signals. 


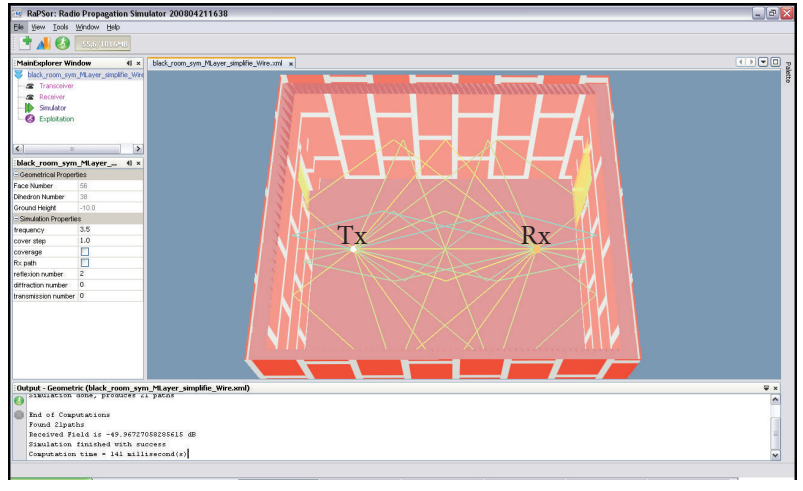

Figure 13: Geometrical paths between Tx and Rx for 2 reflexions and 0 diffraction in the room (cf. Figure 8).

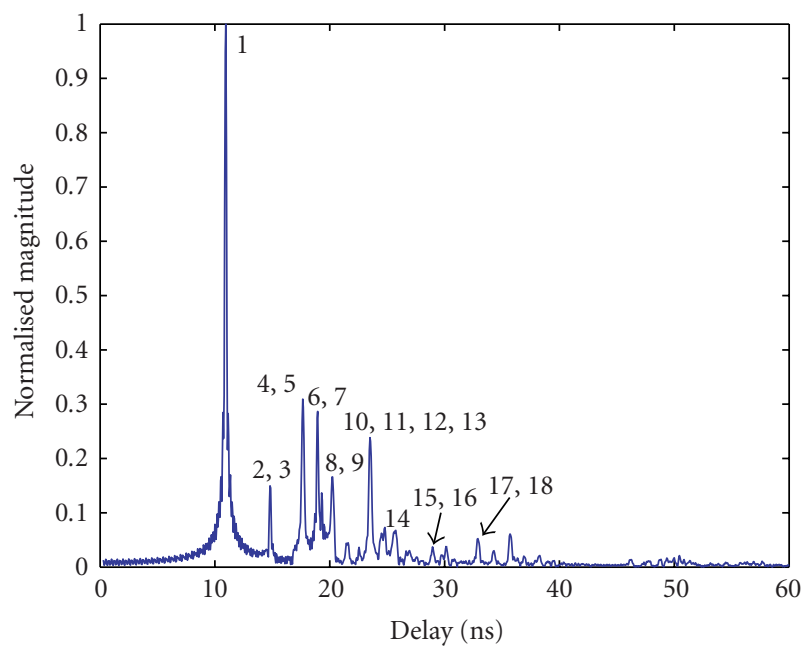

FIGURE 14: Impulse Response of the symmetric case described on Figure 8 .

Thus, we can directly compare simulated and measured signals.

4.3. Echo Analysis. In Figure 12(d), one can observe many echoes with different delays and magnitudes, each of them corresponding to a specific propagation path between the transmitter and the receiver. Figure 13 illustrates all these paths in the test environment of Figure 8.

Due to the width of the emitted wave form (about 5 nanosecond, cf. Figure 9(a)), it is difficult to strictly identify the path delays from the received signal of Figure 12(d). Indeed, the maximum magnitude of the used wave form appears about 2 nanosecond after its transmission beginning, as we can see in Figure 9(a). So there is a time shift between the path arrival and the maximum of the corresponding echo form of about 2 nanosecond. To solve this problem, we propose to directly work on channel impulse response $h(t)$ presented in Figure 14, which is obtained by IFFT of $H(f)$ (cf. Figure 12(b)).
On this curve, the different echoes of the emitted pulse, corresponding to contributions of the paths followed by the waves, have been numbered. We are now going to identify them by analysing the geometrical result of ray tracing algorithm.

As we have seen in Section 2.3, this last one provides the detailed trajectory of each path, especially its length. From here, one can easily deduce the time delay of each path knowing the propagation speed of electromagnetic wave in the air (about $3 \cdot 10^{8} \mathrm{~m} \cdot \mathrm{s}^{-1}$ ). Thus, we are able to make the correspondence between a specific echo in Figure 14 and a specific path between the transmitter and the receiver (cf. Figure 13). As an example and to illustrate the previous comments, we are now going to explain the origin of the 18 echoes presented on Figure 14.

Echo number one being the least delayed, it obviously corresponds to the line of sight path. Then echo noted (2, 3 ) is associated to the reflections on the ground and ceiling, respectively, which are separated by only 0.2 nanosecond. Echo number $(4,5)$ corresponds in fact to two different paths having the same time delay, as illustrated in Figure 15.

The first one (path 4) is reflected on the wall behind the transmitter and then continues until it reaches the receiver (cf. Figure 15(a)). The second one (path 5) is reflected on the opposite wall (behind the receiver) and then reaches it (cf. Figure 15(b)). The fact that they have the same delay is due to the symmetry characteristics of the studied environment. Echoes $(6,7)$ correspond to two symmetric reflected paths as it is shown in Figure 16(a).

Then echoes $(8,9)$ are still associated to two symmetric paths, so identically delayed, corresponding to a first reflection on one of the two side walls as in Figure 15, followed by a second reflection on the ceiling. Echoes (10, 11, 12, 13) are linked to four twice reflected paths as it is shown in Figure 16(b). Once again, for symmetry reasons, these four paths present the same time delay. Echo (14) is associated to two twice reflected paths on the side wall and follows the line (TxRx). Thus they still present the same time delay. Echoes $(15,16)$ correspond to two paths having reflected three times as it is shown on Figure 17(a). Finally, echoes $(17,18)$ are linked to two twice reflected paths of again the same time delay as illustrated on Figure 17(b).

In the next section, we first present a method to highlight, on measured signals, the different contributions which have been presented in this section. Then we confront the measured and simulated signals and discuss on our performance simulator.

\section{Simulation versus Measurement}

To detect an echo due to a reflection on the wall (or floor, or ceiling), we developed a method to highlight the echoes. We placed a metallic plane of $40 \mathrm{~cm}^{2}$ in different positions on the wall, floor or ceiling, to increase the reflection coefficient. We measured the pulse response of the room with and without the metallic plan. We then did a subtraction between these two results. If we obtain a result close to zero, we can consider that there is no reflection on the position of the metallic plan, 


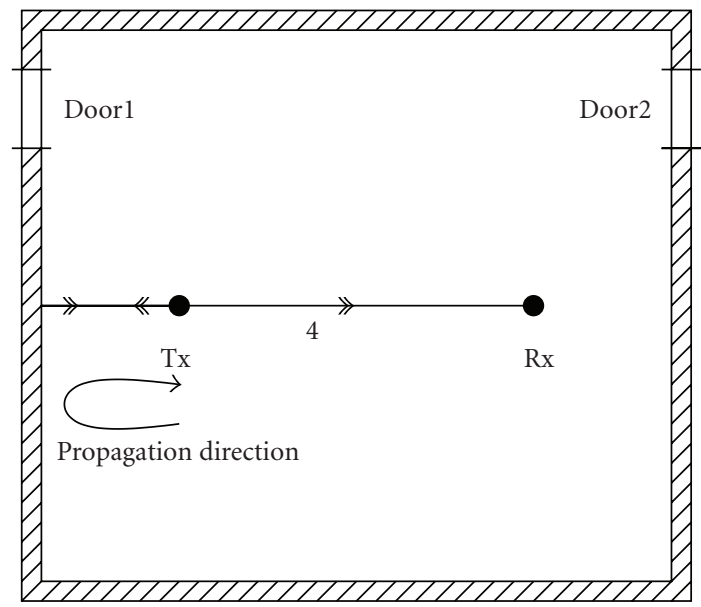

(a)

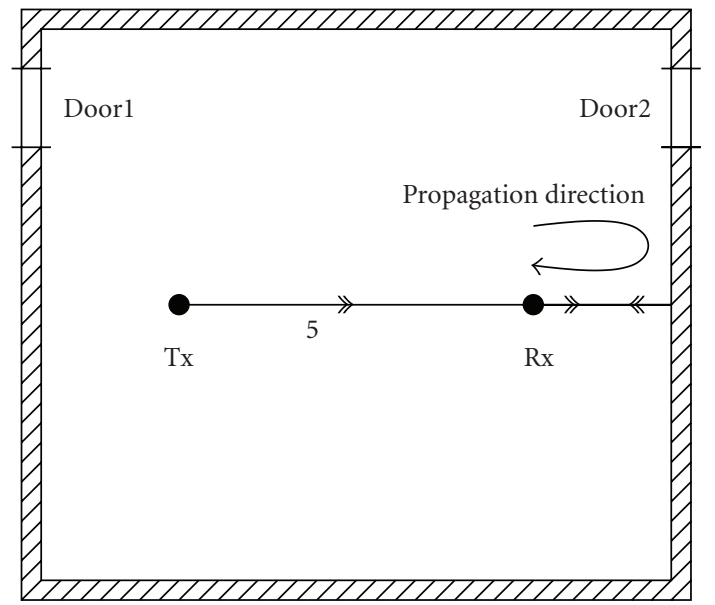

(b)

FIGURE 15: Illustration of paths 4 (a) 5 (b).

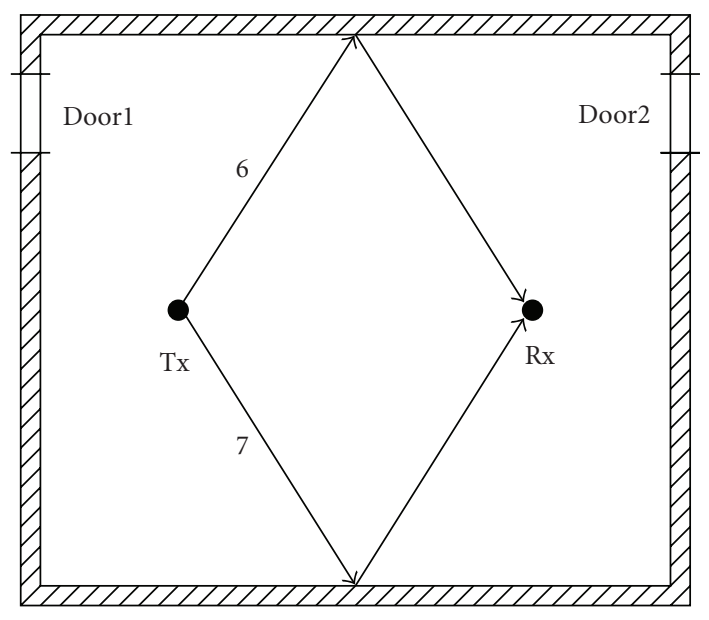

(a)

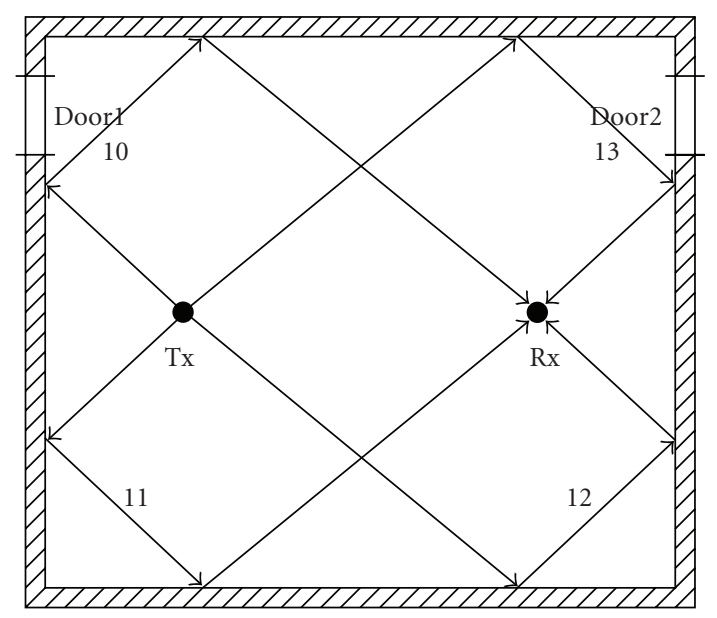

(b)

Figure 16: Illustration of paths (a) 6 and 7 (b) 10, 11, 12, and 13.

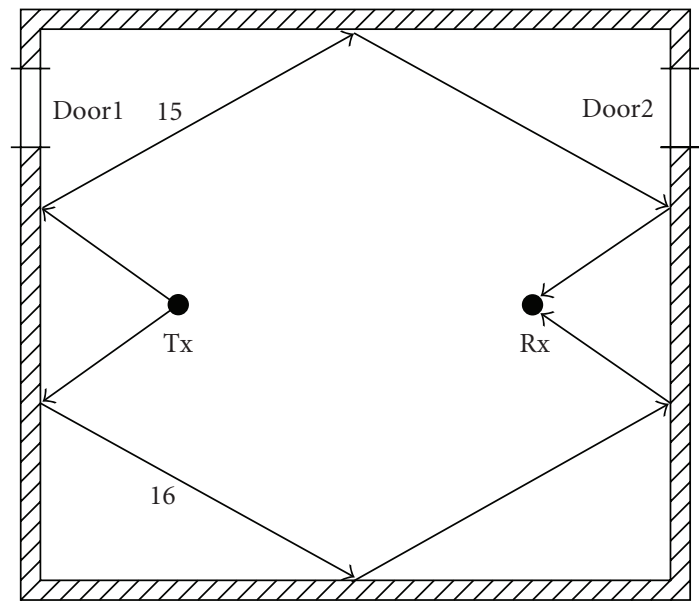

(a)

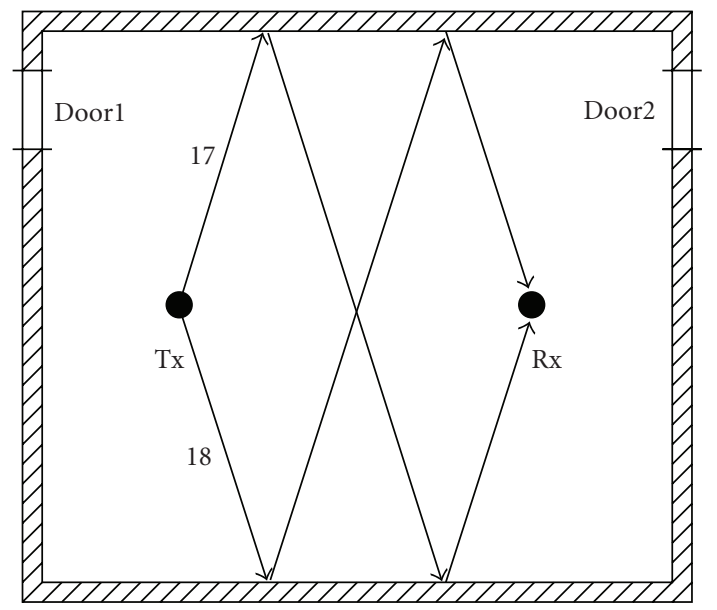

(b)

Figure 17: Illustration of paths (a) 15 and 16 (b) 17 and 18. 


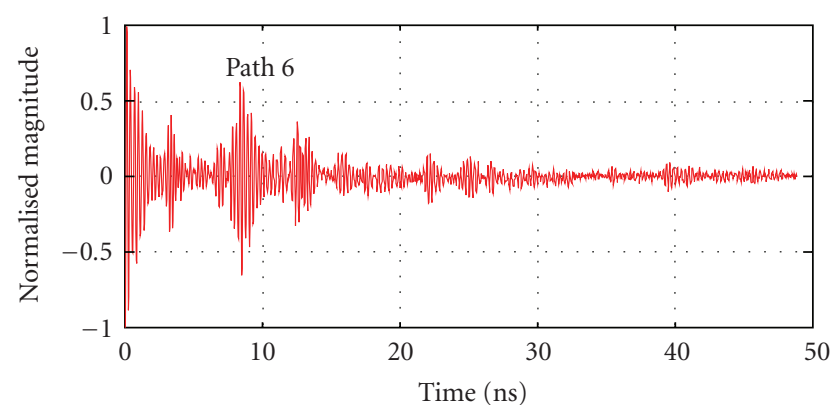

(a)

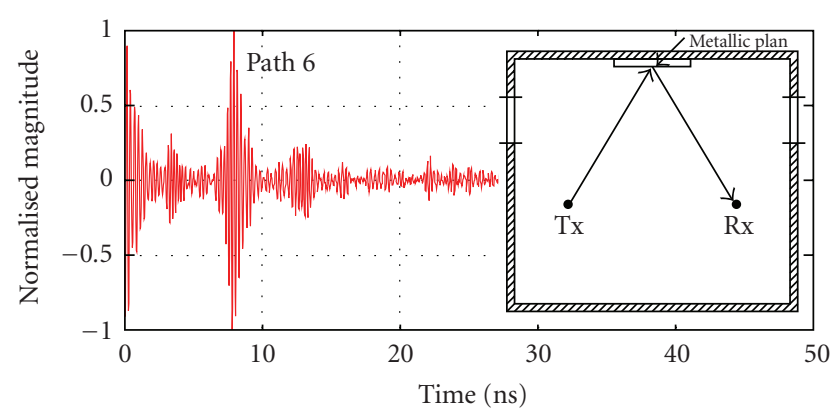

(b)

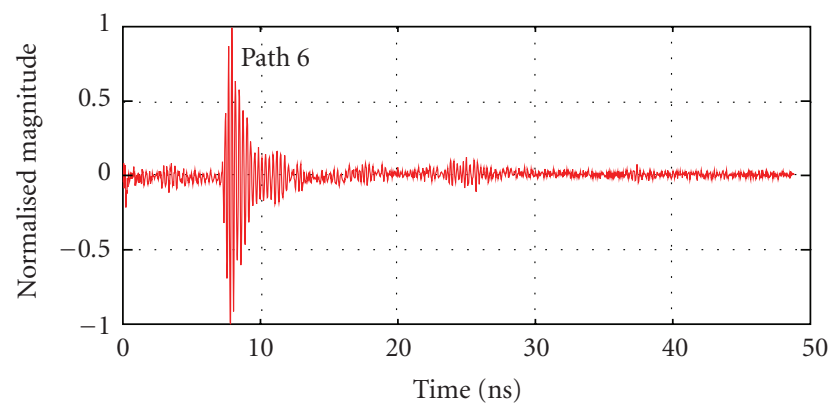

(c)

FIGURE 18: Pointed echo by MTI technique: subtraction between the pulse response of the room (a) and a pulse response (b) with metallic plan put in the way labelled 6 (cf. Figure 16) to increase the reflection phenomenon.

but if we obtain a peak, we can consider that the signal has been reflected on the metallic plan, and also it proves that the pulse has been reflected on the wall.

This method is illustrated by the results in Figure 18, where we have highlighted the path 6 . Figure $18(\mathrm{a})$ is the pulse response of the room. Figure $18(\mathrm{~b})$ is the pulse response of the room, where we have increased the coefficient reflection on the wall for path 6 by putting a metallic plan at the reflection point (cf. room configuration, inside Figure 18(b)). Figure 18(c) is the result of the subtraction of these two acquisitions. All other reflections are cancelled, only path 6 is highlighted (broadcast of path 6 in Figure 18(c) is explained below).

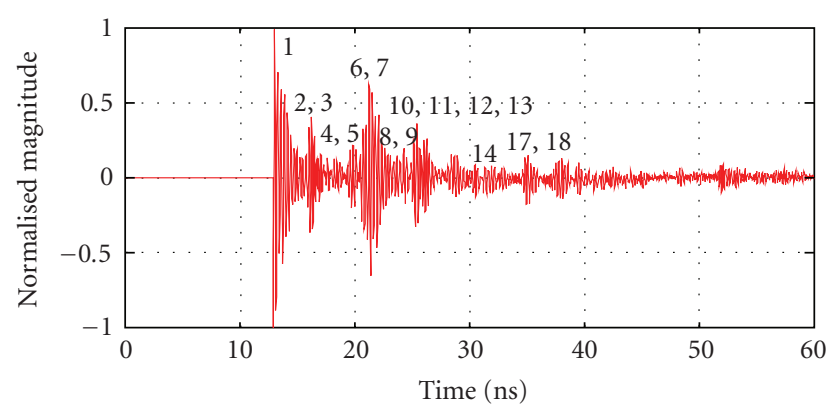

(a)

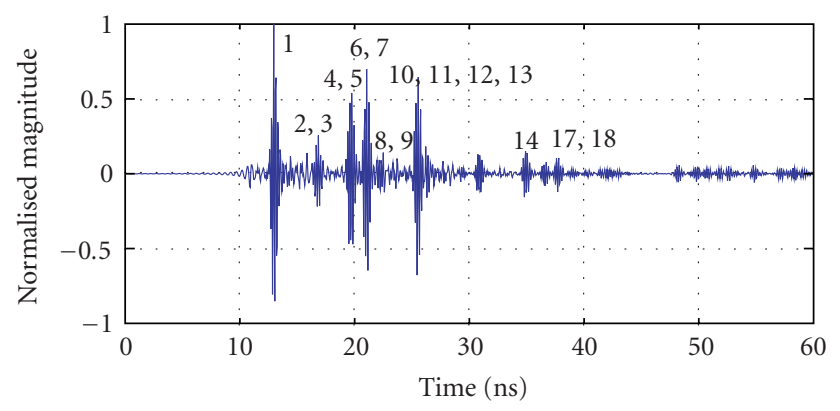

(b)

Figure 19: Comparison between simulation (b) and experiment (a). Echoes are corresponding in symmetric configuration.

The simulated and measured pulse responses of the room (cf. configuration in Figure 8) are compared in Figure 19. Signals are corresponding in time. Each echo is referenced in Figure 19 and corresponds to different paths described in Figures 16 to 17 . Little delays appear sometimes due to the mistakes of acquisition materials.

There is a difference between echoes Figure 19(a), and echoes in Figure 19(b). Echoes in Figure 19(b) are spreading in time. This effect is due to the property of the wall which is composed of multilayered concrete materials, this fact generates a multipath reflection and diffusion effect in the wall: this characteristic causes the phenomena of "broadcasting".

Nonsymmetric ( Tx and $\mathrm{Rx}$ ) configurations are also tested in this room. Simulation and experimental measurements are presented in Figure 20 ( $\mathrm{Tx} / \mathrm{Rx}$ configuration is described in Figure 20). These pulse responses are very similar. Different echoes are labelled in Figure 20 (we have not identified each path, but it is possible with a schematic figure as Figure 13). Figures 19 and 20 show that the simulator works for a UWB propagation signal in a room. The last section describes a radar configuration for a "see through the wall" application.

\section{Through-the-Wall Radar Imaging}

Figure 21 presents the configuration of the Through-theWall Radar Imaging (TWRI) test. We consider the environment of Figure 8 in which we have placed a target of $1 \times 0.01 \times 0.5 \mathrm{~m}^{3}$ at a distance of 1.5 meter from the top wall. Transceiver and receiver are located one above the 


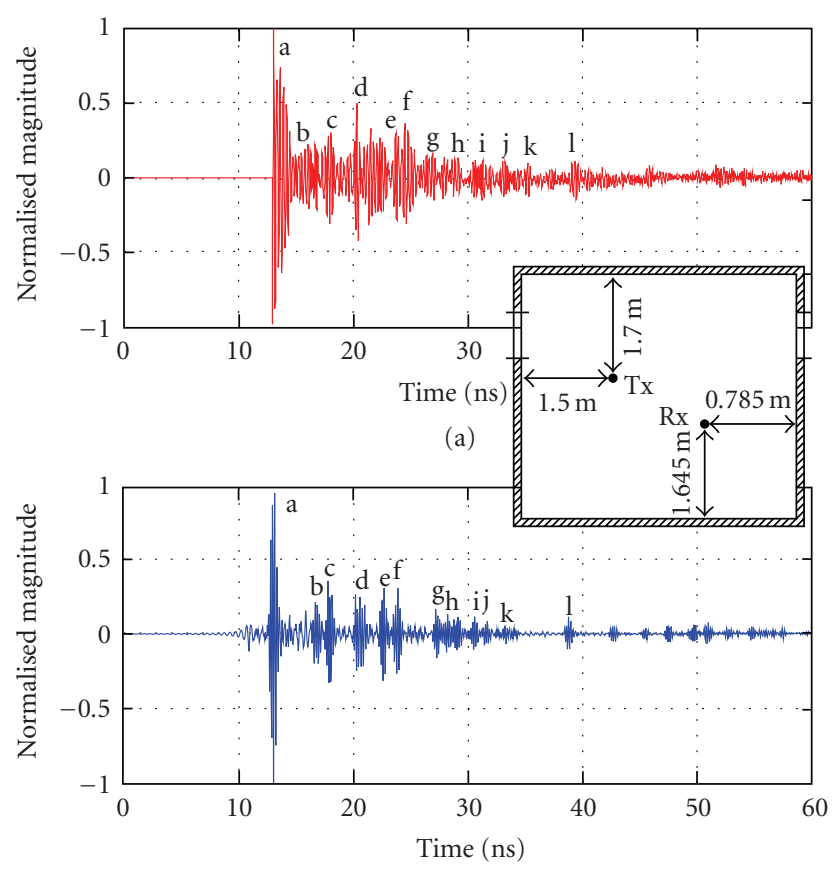

(b)

Figure 20: Comparison between simulation (b) and experiment (a). Echoes are corresponding in asymmetric configuration.

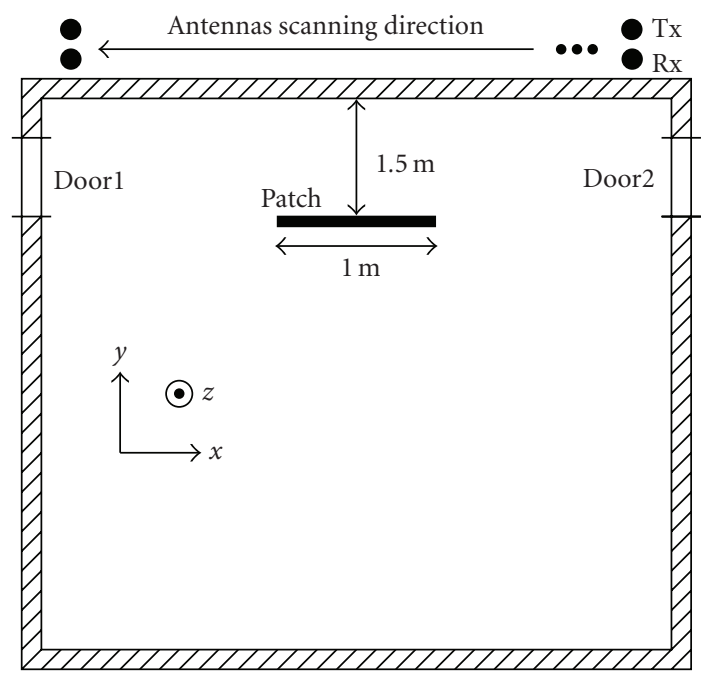

FIgURE 21: Considered configuration for the TWRI test.

other and attached to the top wall in order to eliminate the waves reflected from it. The considered transceiver is quasiomnidirectionnal in azimuth plane whereas the receiver is selective and mainly radiates in the direction of the wall. The half power beamwidth of the receiver antenna is equal to $45^{\circ}$.

Two target configurations have been tested, one considering a perfect electrical conductor in the first time, and another considering a dielectric one in a second instance. In all cases, the experiment consists of simulating the time response of the room with our propagation simulator (cf. Section 2) for each location of the transceiver-receiver couple, this last one moving along the top wall with a fivecentimetre step. Then, we pile these time responses and thus obtain a matrix of which each line represents the room temporal response according to a specified location of the antennas couple. In order to get a good visual rendering of the detected obstacles, each temporal response is loped, and finally we obtain the room images presented in Figure 22.

Echo number 1 obviously corresponds to the wall followed by the antennas couple. Echo number 4 is connected to the room's far end wall. It is important to notice that echo 5 does not appear according to a metallic target because of the quasinull transmission coefficient on such a material, whereas it is present in the case of the dielectric target. Then echo number 2 appearing at a tenth of nanosecond is due to the waves reflected on the target. Finally, echo number 3 is a virtual detection (ghost) of the target. It is due to multiple reflections between the wall near the radar and target (see Figure 23). Behind the dielectric target, the echo of the wall still appears; then it is no longer present in the case of a metallic target: this phenomenon is explained by the fact that some energy, and more, is reflected from the dielectric target, the tie, and reach the wall, unlike the metal target considered as an electromagnetic "mirror," which cannot be crossed (or almost not) by a wave.

\section{Conclusion}

This paper presents the RAPSOR ray tracing simulator originally designed for the propagation of narrowband signals. We have modified this simulator to achieve the UWB propagation. The experiment room was modelled and simulated with UWB pulses. A system to highlight paths on a wall using a metal plane has been developed. We compared the results of simulation with the results of propagation experiments under the same conditions. Different configurations were tested; the results show a good likeness in time. Thus, the simulator is validated to achieve the UWB propagation. A simulator using the method of ray tracing allow obtaining of quick and realistic results. A FDTD simulation method would use much more intensive computation time. Our first images simulated with radar configuration allows us to identify the walls, floor, and ceiling. UWB pulse is too long to discriminate instinctively the shape of the experiment room. To extract all of the room geometrical characteristics, we can use a shorter pulse or use the PDP. This simulator enables to choose radar imaging characteristics for specific rooms: frequency, band, antenna parameters, and so forth. Finally, these images help to clarify ambiguities, which appear on the images produced experimentally, such as ghost echoes.

\section{Acknowledgments}

This work was partly funded by the "Pôle Régional de Recherche en Images, Données et Systèmes" in the PoitouCharentes region, and by the "Plan Pluri-Formations 


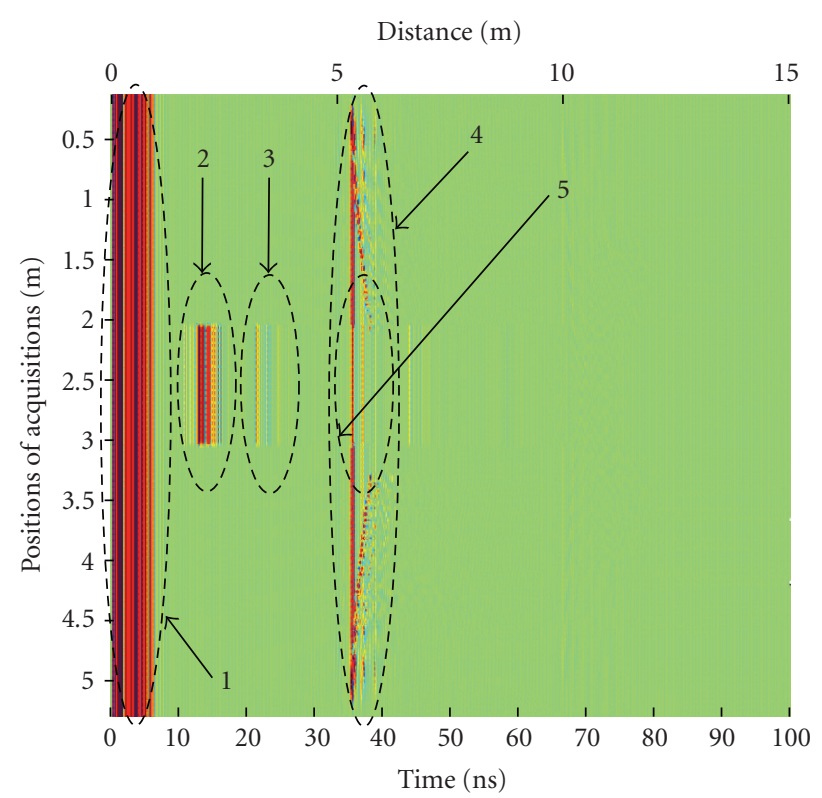

(a)

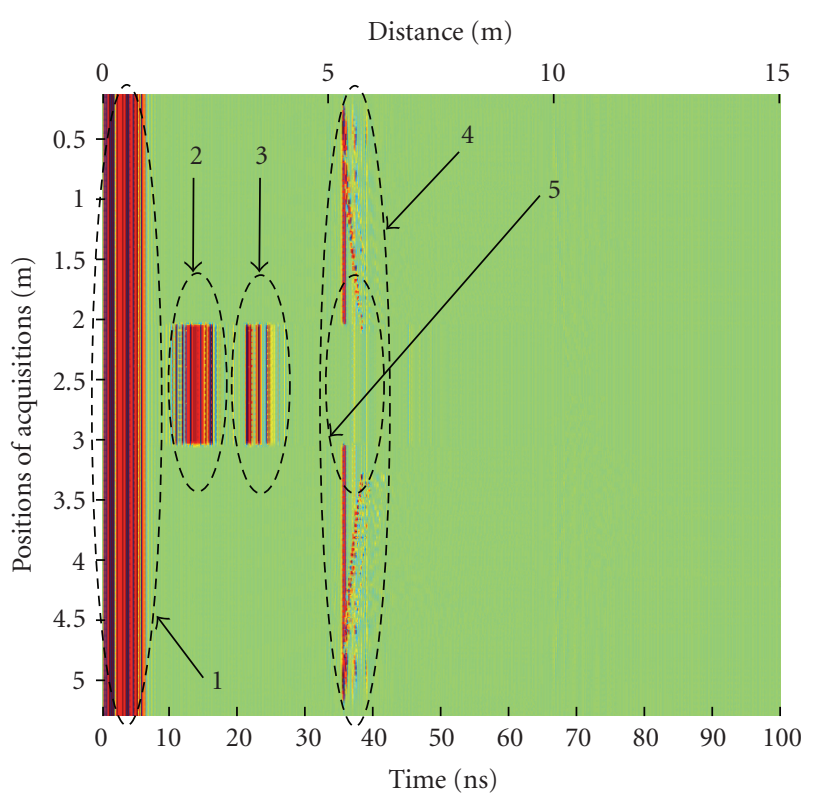

(b)

FIGURE 22: TWRI results in the configuration in Figure 21 according to (a) a metallic target and (b) a dielectric target.

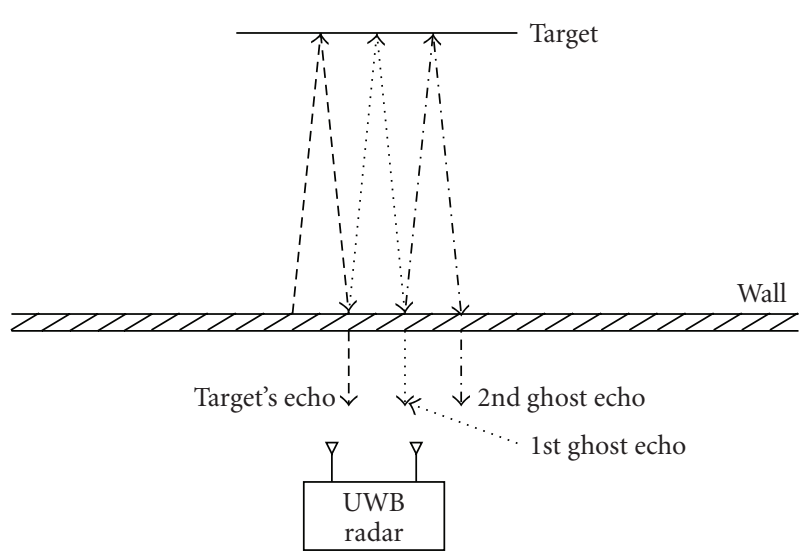

FIgURE 23: Construction of ghost echoes.

Géométrie Images Communications" of the CentreAtlantique PRES (Pôles de Recherche et d'Enseignement Supérieur) in the Poitou-Charente region. The authors would like to thank Mr. M. Tardieu from The University of La Rochelle for mechanical realisation.

\section{References}

[1] S. Nag, M. A. Barnes, T. Payment, and G. Holladay, "Ultrawideband through-wall radar for detecting the motion of people in real time," in Radar Sensor Technology and Data Visualization, vol. 4744 of Proceedings of SPIE, pp. 48-57, Orlando, Fla, USA, April 2002.
[2] C. Liebe, A. Gaugue, J. Khamlichi, M. Menard, and J. M. Ogier, "Radar UWB: vision through a wall," in Ultrawide Band ShortPulse Electromagnetics 9, Springer, New York, NY, USA, 2009.

[3] S. S. Gauthier and W. Chamma, "Surveillance through concrete walls," in Sensors, and Command, Control, Communications, and Intelligence (C3I) Technologies for Homeland Security and Homeland Defense III, vol. 5403 of Proceedings of SPIE, pp. 597-608, Orlando, Fla, USA, April 2004.

[4] M. F. Iskander and Z. Yun, "Propagation prediction models for wireless communication systems," IEEE Transactions on Microwave Theory and Techniques, vol. 50, no. 3, pp. 662-673, 2002.

[5] P. J. Shargo and J. W. Melody, "Model-based correction of through-wall SAR imagery via raytracing," in IEEE Radar Conference, pp. 706-711, April 2007.

[6] P. Combeau, L. Aveneau, R. Vauzelle, and C. Chatellier, "Deterministic propagation model influence on a wireless digital transmission simulation in real environment," in Proceedings of the 15th IEEE International Symposium on Personal, Indoor and Mobile Radio Communications (PIMRC '04), vol. 4, pp. 2421-2425, Barcelona, Spain, September 2004.

[7] L. Aveneau, Y. Pousset, R. Vauzelle, and M. Mériaux, "Development and evaluations of physical and computer optimizations for the UTD 3d model," in Millennium Conference on Antennas \& Propagation (AP '00), Davos, Switzerland, April 2000.

[8] R. G. Kouyoumjian and P. H. Pathak, "A uniform geometrical theory of diffraction for an edge in a perfectly conducting surface," Proceedings of the IEEE, vol. 62, no. 11, pp. 14481461, 1974.

[9] K. Sato, T. Manabe, T. Ihara et al., "Measurements of reflection and transmission characteristics of interior structures of office building in the 60-GHz band," IEEE Transactions on Antennas and Propagation, vol. 45, no. 12, pp. 1783-1792, 1997. 
[10] C. A. Balanis, Advanced Engineering Electromagnetics, John Wiley \& Sons, New York, NY, USA, 1989.

[11] R. J. Luebbers, "Heuristic UTD slope diffraction coefficient for rough lossy wedges," IEEE Transactions on Antennas and Propagation, vol. 37, no. 2, pp. 206-211, 1989.

[12] K. Siwiak, "An introduction to ultra-wideband wireless technology," in Proceedings of the 53rd IEEE Vehicular Technology Conference (VTC '01), Rhodes, Greece, May 2001. 

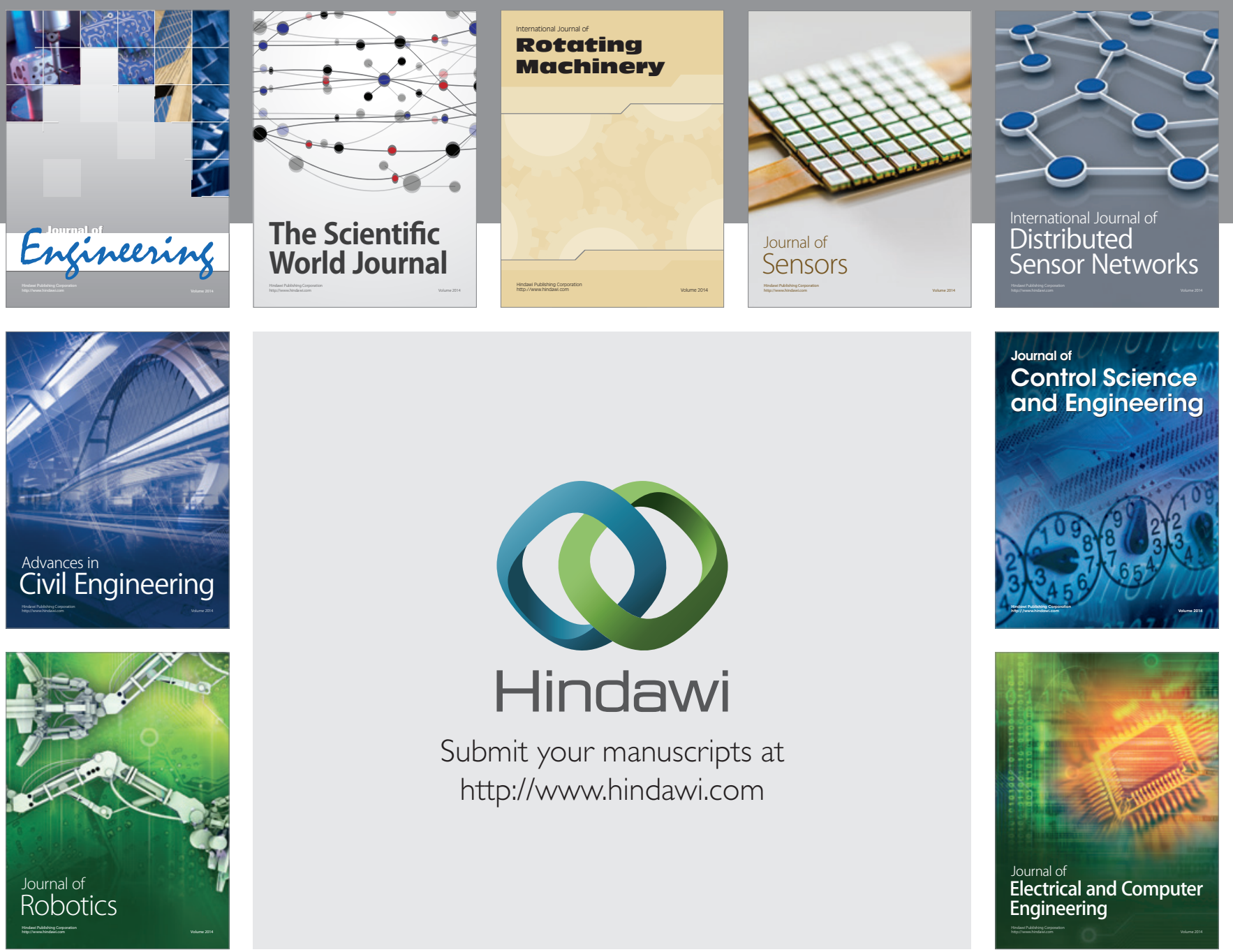

Submit your manuscripts at

http://www.hindawi.com
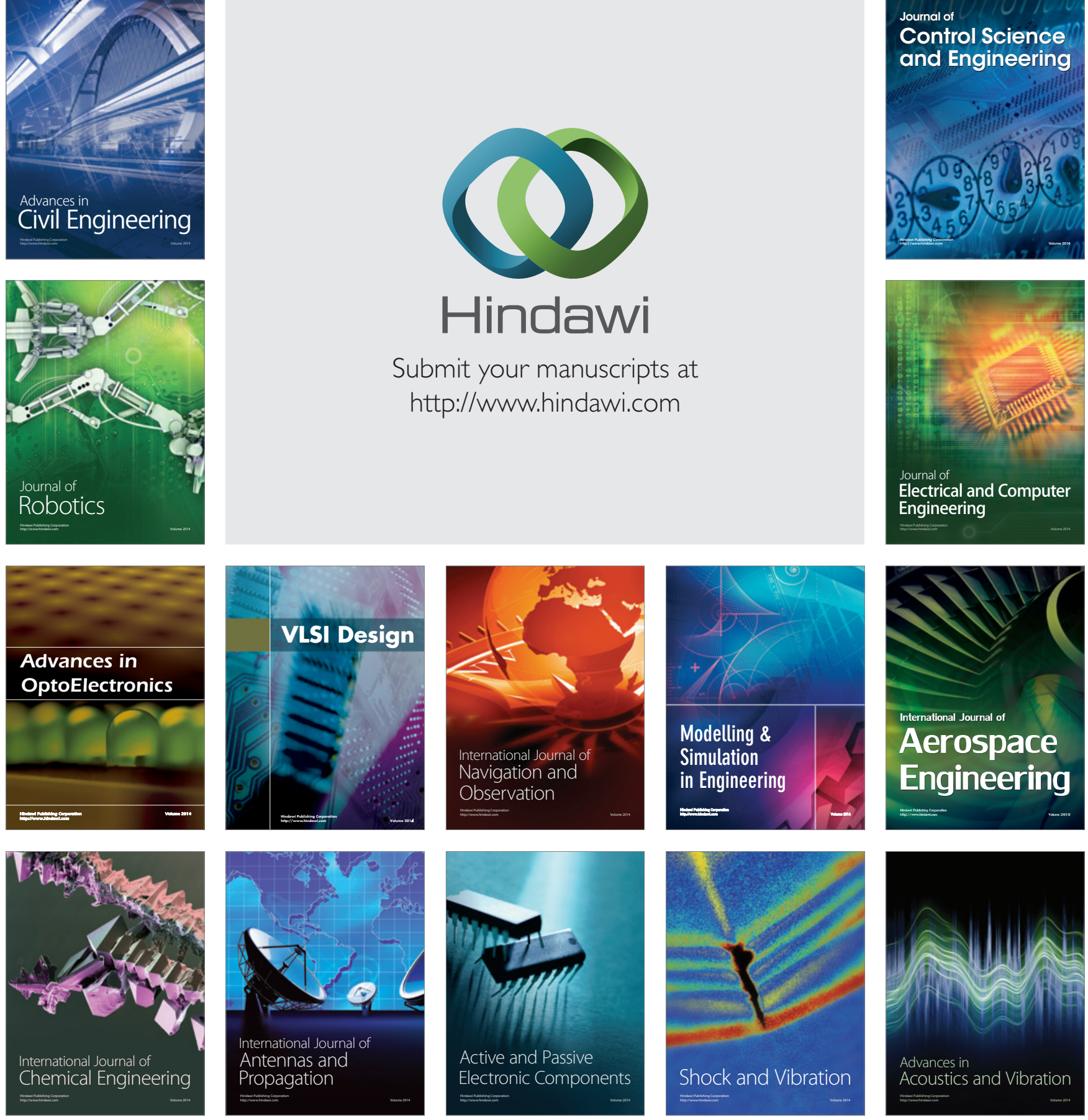